\title{
Comprehensive Technologies for Iron Ore Sintering with a Bed Height of 1000 mm to Improve Sinter Quality, Enhance Productivity and Reduce Fuel Consumption
}

\author{
Zhengjian LIU, ${ }^{1)}$ Lele NIU, ${ }^{1)}$ Shijun ZHANG ${ }^{2)}$ Guanqiu DONG ${ }^{21}$ Yaozu WANG, ${ }^{1)}$ Guilin WANG ${ }^{1)}$ Jian KANG, ${ }^{1)}$ \\ Longzhi $\mathrm{CHEN}^{11}$ and Jianliang ZHANG ${ }^{1,3) *}$ \\ 1) School of Metallurgical and Ecological Engineering, University of Science and Technology Beijing, Beijing, 100083 China. \\ 2) Tiangang United Special Steel Co., Ltd., Tianjin, 301500 China. \\ 3) School of Chemical Engineering, The University of Queensland, St Lucia, QLD, 4072 Australia.
}

(Received on April 10, 2020; accepted on July 1, 2020)

\begin{abstract}
Despite its many advantages, the materials layer with thickness exceeding $900 \mathrm{~mm}$ hinders further development of thick bed sintering. In order to break through the bottleneck of ultra-thick bed sintering, University of Science and Technology Beijing and Tiangang United Special Steel initiated a cooperation. Firstly, by analyzing the characteristics of the ultra-thick bed sintering, this study aims to make high air permeability and low air leakage rate the focus technically. Then a "triple sync" theory including the liquid phase front, heat transfer front and flame front and the concept of "Full Active Lime Intensified Sintering" were proposed to support thick bed sintering. On this basis, Tiangang United Special Steel develops a series of comprehensive technologies including raw materials controls, granulation enhancements and air leakage improvements, then efficient and stable sintering production with a bed height of $1000 \mathrm{~mm}$ in their two $260 \mathrm{~m}^{2}$ sintering machines is achieved. After the implementation of these technologies, the productivity reaches $1.89 \mathrm{t} /\left(\mathrm{m}^{2} \cdot \mathrm{h}\right)$, the solid fuel consumption was only $41.85 \mathrm{~kg} / \mathrm{t}$, meanwhile the tumbler, reduction, and low-temperature reduction degradation index $\mathrm{RDI}_{+3.15}$ of produced sinter are $78.24 \%$, $87.17 \%$, and $74.2 \%$, respectively. While promoting the development of ultra-thick bed sintering, it has also brought significant economic and environmental benefits.
\end{abstract}

KEY WORDS: comprehensive technologies; ultra-thick bed sintering; air permeability; air leakage; full active quicklime.

\section{Introduction}

Thick-bed sintering can take advantage of the self-storage effects of the materials layer to lower the consumption of solid fuels, reduce the emission of gaseous pollutants such as $\mathrm{NO}_{x}, \mathrm{CO}_{x}, \mathrm{SO}_{x}$, etc., while increasing the output of the sintering process and improving the sinter strength. ${ }^{1-3)}$ Since the $1950 \mathrm{~s}$, it has been the consensus for steel enterprises around the world to optimize the iron ore sintering production by increasing the thickness of materials layer. As a result materials layer thickness of sintering machines has also continued to increase, from $200-300 \mathrm{~mm}$ at that time to more than $700 \mathrm{~mm}$ now. Due to the limitation of special iron ore resources and technical conditions, later development of thick-bed sintering technology in China results in a thickness of 200-300 mm for a long period compared with Europe and Japan. Since 21st century, thick-bed sintering

\footnotetext{
* Corresponding author: E-mail: zhang.jianliang@hotmail.com
}

begins to develop rapidly all over the country and the average thickness has exceeded $700 \mathrm{~mm}$ in recent several years, as shown in Fig. 1, effectively reducing the energy consumption of Chinese iron ore sintering process. ${ }^{4}$ Meanwhile a few steel enterprises are still devoted to higher and higher layer thickness.

Although thick-bed sintering has been widely applied to improve sinter production and reduce fuel consumption, however some negative effects began to appear with the continuous increment of layer thickness. When the thickness of the material layer is increased, the resistance of material layer is also increased, resulting in a decreased amount of exhaust air and insufficient sintering power, so the heat transfer, mass transfer, and physical-chemical reactions are influenced accordingly. ${ }^{5)}$ In this case, the sintering speed and the productivity will decrease, and the sinter quality will also be uneven. In addition, the thickness of the overwetting zone increased with the thickness of the material layer, which further deteriorates the air permeability of the 


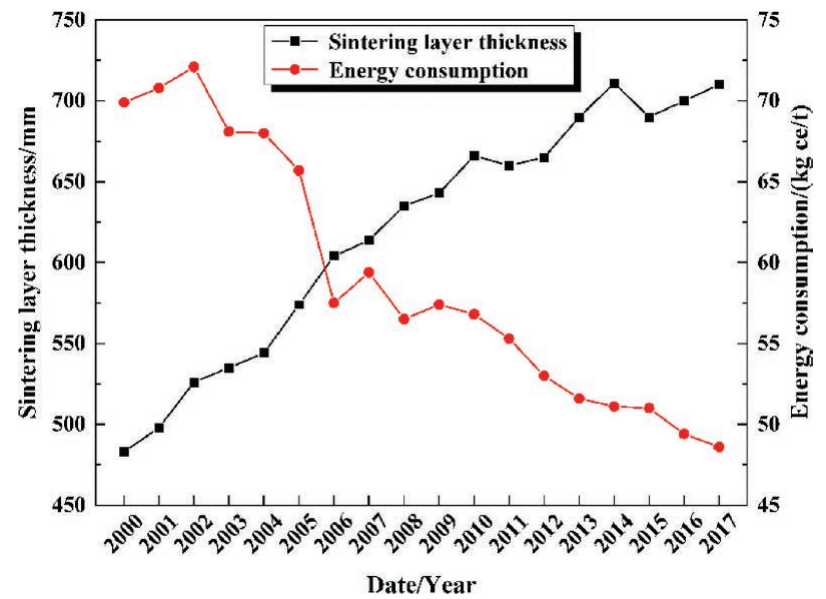

Fig. 1. The relationship between sintering layer thickness and energy consumption in China in recent years. (Online version in color.)

material layer. Without corresponding countermeasures, the advantages of thick-bed sintering are not obvious, but may also have unexpected or opposite effects. Therefore, many researches contributing to new technologies have emerged in the field of thick layer sintering in recent years.

Jiang et al. $^{6-8)}$ proposed the new agglomeration technology of composite agglomeration process (CAP), where magnetite concentrates is prepared into pellets and sintered together with other raw materials. The pellets play a "skeleton" role in the sintering material layer, which can effectively improve the gas permeability and reduce wind resistance of the material layer. In addition, the fuelfree pellets are mostly distributed in the lower position of sintering layer to maximize the self-heat storage effect. In his study, efficient sintering can be achieved in ultra-thick material layer through the CAP. The double-layer sintering method proposed by Nikolaev from the former Soviet Union can also support the sintering production with a high thickness of the material layer. The double-layer feeding and the double-layer ignition could realize the operation of two combustion zones simultaneously, thereby reducing the thickness of the over-wetting zone. A large amount of oxygen-enriched air is need in double-layer sintering method. Anshan Iron and Steel in China currently applies double-layer sintering for production. ${ }^{9)}$ Stand-support sintering is also one developed method for thick-bed sintering by Higuchi et al. ${ }^{10)}$ which uses bars or plates attached to pallets to support sinter cake, lightening the load from the upper part. Their research shows that stand-support sintering can accelerate sintering and improve productivity by $20 \%$. Zuo and Liu et al. ${ }^{11-13)}$ in China also made lots of works on supporting sintering technologies applied into industrial trails, results shows that sinter production with stand-plates exhibit decreased fuel consumption of $1.32 \%$, and an increase in productivity of $6.34 \%$.

However, all the above-mentioned technical methods have employed extra processes or equipments to achieve high-efficiency and stable thick-bed sintering. In actual production, either the equipment cost or the complexity of operation was significantly increased. Through the cooperation with University of Science and Technology Beijing, Tiangang United Special Steel achieved stable and efficient production of two $230 \mathrm{~m}^{2}$ sintering machines with a materials layer thickness of $1000 \mathrm{~mm}$ since 2016 through innovations of technologies and concepts. Many sintering indices of the two sintering machines are all at high-level in China.

\section{Background and Logic of Technologies}

There are two key technical points, namely high air permeability and low air leakage rate, for the practice of ultra-thick bed sintering. Therefore, the focus of comprehensive technologies is to reduce the air leakage rate and achieve high permeability of materials layer.

In terms of reducing the air leakage rate, a series of traditional and innovative methods are applied to optimize the sintering machine in order to achieve the goal of "side-sealing middle-passable". High air permeability is technically realized mainly through strengthening granulation and reducing the exhaust resistance of sintering materials layer. Strengthening granulation requires control of raw material particle size and quasi-particle formation, while optimized control of over-wetting zone and combustion zone is needed to reduce materials layer resistance. ${ }^{14)}$ On this basis, the theory of triple sync is proposed, that is, the concept of liquid-phase front is added based on the traditional heat transfer front and flame front of sintering materials layer. ${ }^{15,16)}$ The high-efficiency and stable sintering with an ultra-thick bed can be achieved only when the three are synchronized. Quicklime has unique advantages in granulation and liquid phase formation, thus exerting its advantages and eliminating its shortcomings during the application, which are linked together to form the "Fully Active Lime Intensified Sintering" technology. As shown in Fig. 2, the logic of the comprehensive technologies is interlocking as described above.

\section{Comprehensive Technologies Implementation}

Figure 3 shows the production flow chart of Tiangang United Special Steel from the raw materials field to sintering machine. The numbers in the figure denote the technical measures and equipment transformation at different positions during the sintering process. Position 1 indicates the spiral lining of the secondary mixing drum, position 2 indicates that the mixture is supplemented with hot water to increase the materials-temperature, position 3 indicates the injection of steam from the sintering machine head, position 4 indicates the transformation of the igniter, positions 5, 6 and 7 shows the key positions of air leakage control. This flow chart provides support for subsequent technical introductions.

\subsection{Raw Materials Controls}

\subsubsection{Iron Concentrates Proportion}

The particle size of the raw materials is directly related to the granulation of the sintering mixture. The number of nuclear particles will be insufficient and the bulk density in the material layer will increase if the particle size of iron ore is too fine, which deteriorates the air permeability of the materials layer, leading to poor sinter quality. ${ }^{17)}$ Chinese sintering plants generally employ local iron concentrates and imported hematite and limonite with larger particle size. 


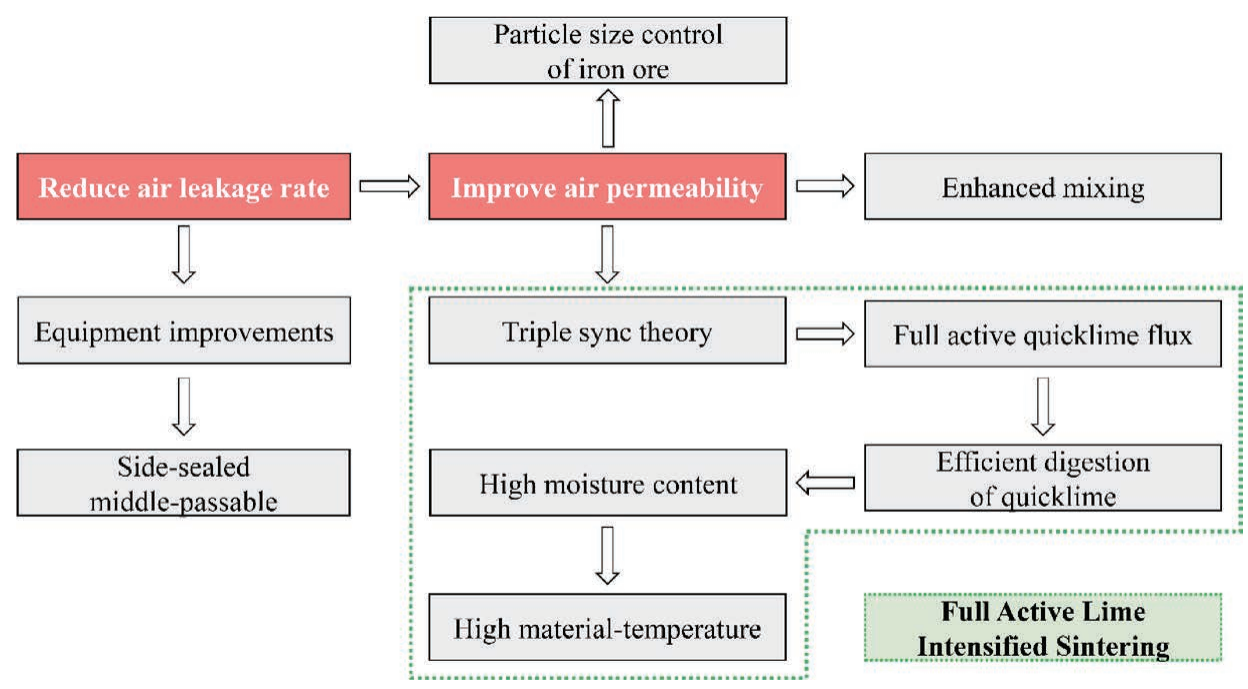

Fig. 2. Logic of the comprehensive technologies. (Online version in color.)

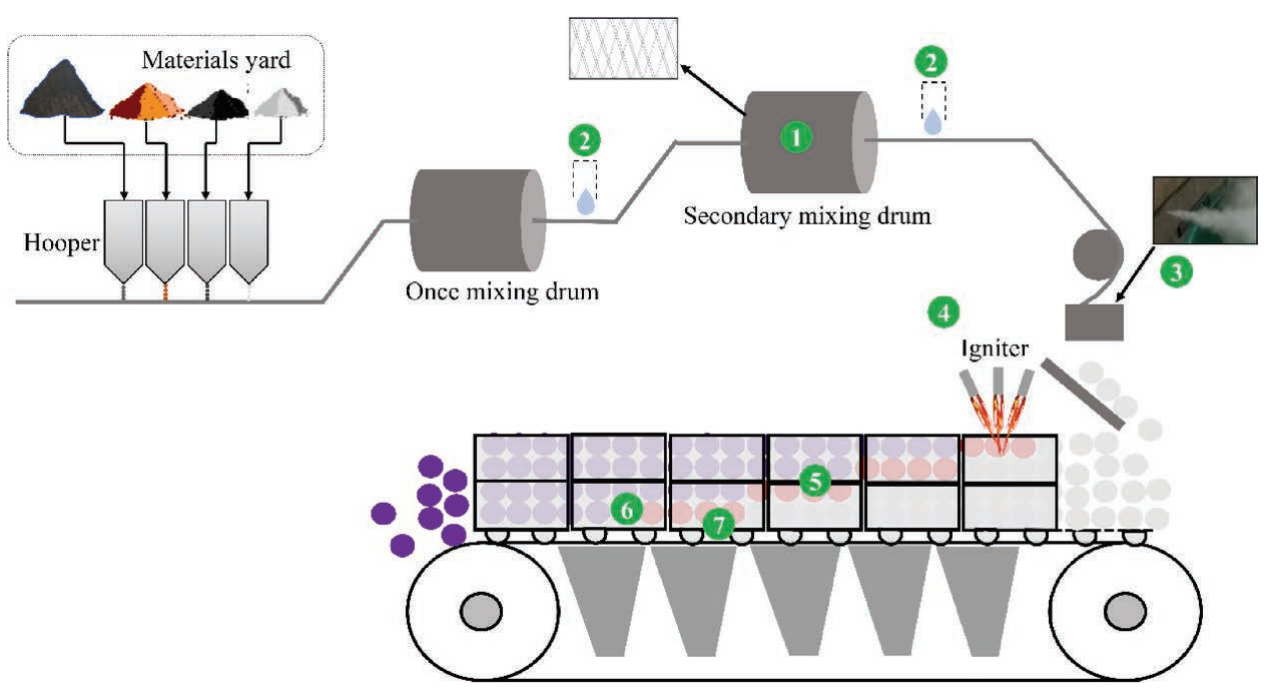

Fig. 3. Flow chart of sintering in Tiangang United Special Steel. (Online version in color.)

The gas permeability of the material layer is limited for the thick-bed sintering, so the characteristic of fine particle-size directly determines the unavailable application in a large proportion for the iron concentrates due to its poor granulation performance. In the production of 1000 $\mathrm{mm}$ thick-bed sintering of Tiangang United Special Steel, the proportion of iron concentrates with a particle size less than $1 \mathrm{~mm}$ is controlled to be below $12 \%$ strictly.

\subsubsection{Flux Structure Selection}

Flux plays an important role in granulation and assimilation during sintering process. The fluxes often selected during sintering process are quicklime, limestone, dolomite, magnesite, etc. In most sinter plants, these fluxes are usually used in conjunction with each other, given their different physical and chemical properties. However, in Tiangang United Special Steel, 100\% quicklime is placed in the flux hopper due to its unique properties, which meets the needs of ultra-thick bed sintering.

One of the reasons is that the ultra-thick bed sintering has higher requirements on the granulation performance of the raw materials mixture. Quicklime has a finer particle size and better dispersion compared with other fluxes, thus it can be uniformly distributed in the material layer during the granulation process, which is beneficial for the formation of liquid phase SFCA during the sintering process, as a result, the sinter consolidation strength and yield is improved. ${ }^{18,19)}$ Secondly, the high material-temperature of the mixture play significant role in eliminating the over-wetting zone in sintering layer and improving the sintering indices. Active quicklime has a absolutely high content of $\mathrm{CaO}$ with released large amount of heat during digestion, which can effectively increase the temperature of the material mixture. In addition, in many previous studies ${ }^{20,21)}$ on blast furnace coal injection and the characteristics of fuel combustion during sintering, the existence of $\mathrm{CaO}$ can promote the combustion of carbon in solid fuel. As the combustion of the sintering flame front is one of the most important factors affecting the sintering process, a large amount of $\mathrm{CaO}$ in quicklime can promote the combustion of pulverized coal and coke breeze, reducing the thickness of the combustion zone, which is the second largest area of exhaust resistance after the over-wetting zone. This contributes to the realization of the advantages of ultra-thick bed sintering. ${ }^{14)}$ In order to take advantage of the merits of quicklime, fluxes of mainly carbonates including limestone and dolomite were completely discarded with the 
proportion of quicklime reaching to $7.6 \%$.

\subsubsection{Activity Control of Quicklime}

The activity of quicklime determines whether it can take full advantage of its performance. Strict activity controls are conducted by Tiangang United Special Steel on the purchased quicklime, including testing whether the quicklime meets the standard by the method stipulated by the Chinese ferrous metallurgy industry standard YB/T 105-2014 and the convenient detection based on actual production. The former requires an activity higher than $300 \mathrm{~mL}$. In more than 3000 tests of 2019, the average $\mathrm{CaO}$ content of the used quicklime was determined to be $86.12 \%$, the average activity was $326.51 \mathrm{~mL}$. The convenient detection is shown in Fig. 4, a $500 \mathrm{~g}$ sample of quicklime is placed into a metalware, then weighing $250 \mathrm{~mL}$ of water into the metalware with a graduated cylinder. At the same time, the timer will start counting. Afte 2 minutes (the travel time of the sintering mixture in a mixing drum), whether the measured quicklime activity is qualified for sintering production is judged based on whether the quicklime in the vessel is completely digested (no more smoke).

\subsection{Granulation Enhancements}

The granulation technologies here is mainly aimed at the technical measurements used in the batching and mixing process. The first is the improvement of the mixing drum. Secondly, the premise that quicklime has a positive effect on the granulation process is the use of large amounts of water for sufficient digestion. Finally, high material-temperature is not only the result of full digestion of quicklime, but also a necessary measure to eliminate the negative effects induced by large moisture. The comprehensive application of these granulation technologies reduced the proportion of the $-1 \mathrm{~mm}$ portion in the mix from nearly $17 \%$ to $14.91 \%$.

\subsubsection{Enhanced Mixing-drum Transformation}

The drum mixing is of great significance for granulation. Generally speaking, the secondary mixing is to make the raw materials into quasi-granular pellets after the first uni-

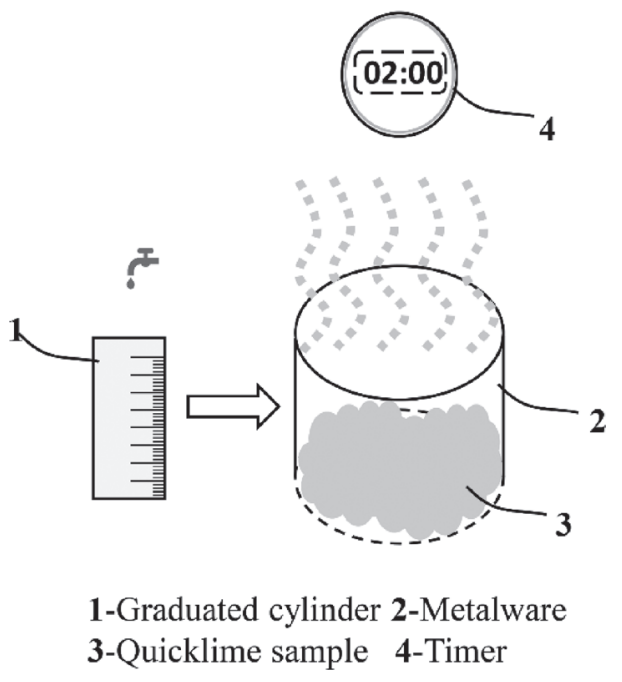

Fig. 4. Schematic diagram of quick detection method for quicklime activity. form mixture of the sintering raw materials occurs. Therefore, the secondary mixing will affect the final granulation performance, meanwhile the air permeability of materials layer is influenced more significant. It is easy to cause the phenomenon of sticking and form a pile at the exit of the drum due to addition of water in the drum, resulting in poor granulation effect and low filling rate. Therefore, Tiangang United Special Steel modified the secondary mixing drum. As shown in Figs. 2 (position 1) and 5, a ceramic tapered counterflow lining plate was installed inside the drum, the spiral direction of the lining plate was set to be opposite to the rotation direction of the drum. The angle steel is fixed with bolts on the countercurrent spiral lining plate in the drum as a bead, and the placement direction of the angle steel bead is adjusted to better lift the material and make the mixture more uniform. The inlet and outlet of the drum is made of respective small and large angle steel, forming a cone shape.

In addition, the tapered counterflow liner extends the path of the sintering mixture in the drum, which is also beneficial for the contribution of the full quicklime flux structure. It is equivalent to indirectly prolonging the digestion time of quicklime and helping the quicklime to fully digest, contributing to the generation of liquid phase during sintering process and avoiding the generation of "white spots" in the sinter.

\subsubsection{High Moisture Content}

In the traditional sintering concept, it is believed that a low water proportion can reduce the solid fuel (carbon) consumption. Therefore, the moisture content for sintering mixture is generally controlled between $6-7.5 \%$ in most Chinese steel enterprises. The sintering mixture in Tiangang United Special Steel possesses the highest moisture content of $9.0 \%$ with averaged of about $8.5 \%$. On the one hand, as the binder of the sintering mixture, a large amount of water can make the sintering materials fully form quasi-particles, increasing the average particle size of the mixture and improving the air permeability of the materials layer. ${ }^{22)}$ The high moisture content influences the particle size range of the adhering fines to a certain extent, so that larger particles can also act as adhering fines during the granulation process. On the other hand, full quicklime can be fully digested by enough water due to its the flux structure, forming a cohesive colloid, which would improve the granulation effect and also contribute to increase the sinter strength.

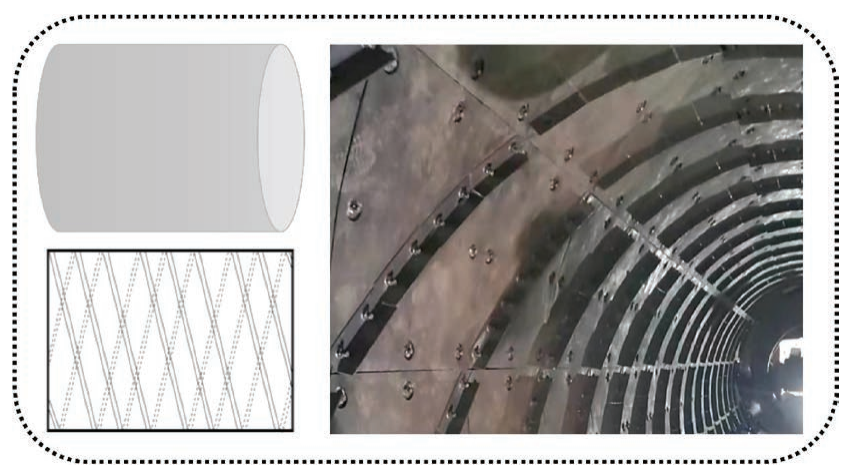

Fig. 5. Schematic of tapered countercurrent mixing drum (Online version in color.) 


\subsubsection{High Material-temperature}

The over-wetting zone as the most important link can restrict the air permeability of the sintering materials layer, and sintering with an ultra-thick bed will increase the thickness of the over-wetting zone. A large amount of water is needed in flux structure of full quicklime, which will further exacerbate the over-wetting property in the lower part of the materials layer. Therefore, a high material-temperature is mandatory in order to achieve high air permeability of the materials layer. At the material-temperature higher than dew point temperature, the moisture in the materials layer will not condense into liquid water, which can effectively reduce or even eliminate the over-wetting zone. ${ }^{23)}$

The moisture content of the exhaust gas in the lower part of the sintering materials layer can be calculated by Eq. (1), and then the dew point temperature in the lower part of the materials layer can be obtained by comparing the saturated vapor pressure table.

$$
\theta=\frac{L \times d \times v \times \rho \times w}{Q \times(1-f)} \times P+w^{\prime}
$$

Where $\theta$ represents the moisture content of the exhaust gas in lower part of the materials layer $\left(\mathrm{g} / \mathrm{m}^{3}\right) ; L$ is the width of sintering pallet $(\mathrm{m}) ; d$ is the height of sintering materials layer $(\mathrm{m}) ; v$ is speed of sintering machine $(\mathrm{m} / \mathrm{min}) ; \rho$ is the bulk density of sintering mixture $\left(\mathrm{t} / \mathrm{m}^{3}\right) ; w$ is the moisture content of sintering mixture $\left(\mathrm{g} / \mathrm{m}^{3}\right)$; $\mathrm{Q}$ is the air extraction of the fan $\left(\mathrm{m}^{3} / \mathrm{min}\right) ; f$ is the air leakage rate of sintering machine $(\%)$; $P$ is the exhaust pressure (atmospheric pressure); $w^{\prime}$ is the atmospheric humidity $\left(\mathrm{g} / \mathrm{m}^{3}\right)$.

According to the related data, the moisture content of the exhaust gas in Tiangang United Special Steel is determined to be $139.3 \mathrm{~g} / \mathrm{m}^{3}$, and the corresponding dew point temperature in the saturated vapor pressure table is $62^{\circ} \mathrm{C}$.

The full active quicklime flux with large moisture content can make full use of the heat generated by quicklime digestion to increase the material-temperature. In addition, Tiangang United Special Steel adopted the measurements of raising temperature in the full sintering process, including: (1) the mixing drum sprays superheated steam recovered from the flue; (2) the sintering machine head sprays superheated steam and saturated steam generated by denitration waste heat (as shown in Fig. 3 position); (3) heating water used for once mixing with saturated steam; (4) replenishing hot water during belt transmission (as shown in Fig. 3 position 2). It is worth mentioning that superheated steam can increase the material-temperature without appearance of moisture. Based on these measurements, the materialstemperature is stabilized at $76-82^{\circ} \mathrm{C}$ (above dew point -temperature), indicating that no an over-wetting zone in the lower part of materials layer is achieved and the air permeability of materials layer is greatly improved.

\subsection{Air Leakage Improvements}

"Side-sealed middle-passable" has always been the key to address air leakage and improve sintering production efficiency. Tiangang United Special Steel has conducted a bunch of innovative equipment modifications except for conventional solutions, which effectively reduced the air leakage rate of the sintering machine from $55 \%$ to $34.68 \%$. Correspondingly the power consumption of the fan is also reduced dramatically. Figure $\mathbf{6}$ shows the main equipment modifications to reduce the air leakage rate.

As the thickness of the materials layer increases, gap at the joint of the trolley rail is always observed due to the effect of materials extrusion (as shown in position 5 of Fig. 3). Tiangang United Special Steel uses a single side rail combination device to make the three upper and lower rail plates consolidate well. In addition, a triangular bolt arrangement is used to fasten any two rail plates, preventing the rail from tilting and separating efficiently.

The sintering material is distributed on the pallet grate, the pressure block holding the grate are usually fixed on the pallet rails by pin rod. There is a gap between the pin rod and the pin hole on the pallet rail, leading to instability of the pin rod installation. With the movement of the pallet and the effect of air extraction, air leakage will be induced by this gap at the position of 6 in Fig. 3. Therefore, the pin rod of the new pressure block structure includes a proximal polished rod and a distal threaded rod. On the threaded rod, a circumferential sealing gasket and a circumferential pressing piece are sequentially set along outside of the rail, which are bolted on rail. The application of this pressure block structure can eliminate about $3 \mathrm{~mm}$ gap between the pin rod and the pin hole, which has a great effect on reducing air leakage rate for a pallet with many pressure blocks.

Although the sealing structure of the lower part of pallet (as shown in position 7 of Fig. 3) is important in controlling air leakage, the lower part of the pallet is always the main location responsible for air leakage. The sealing structure is generally composed of a static oil plate, a movable oil plate and an upper groove plate. Springs are uniformly arranged

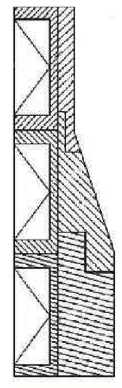

5

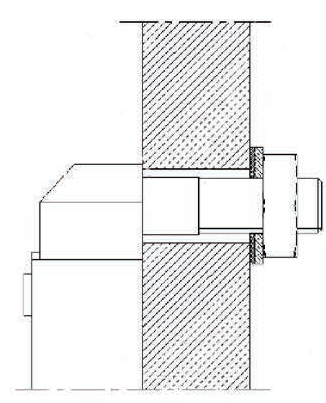

6
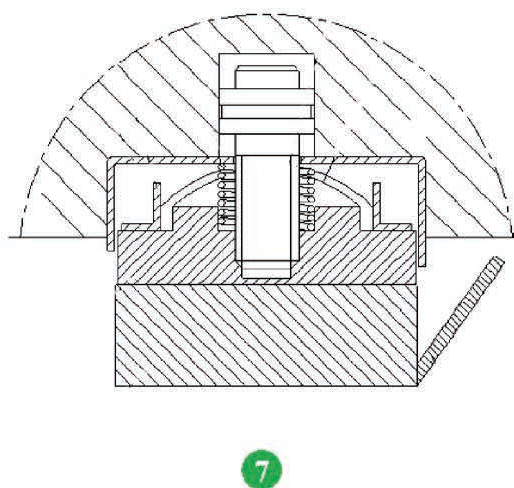

Fig. 6. Schematic diagram of equipment transformation for air leakage. (Online version in color.) 
along the extending direction between the movable oil plate and the upper groove plate. The static oil plate is arranged below the movable oil plate, combining with moving oil plate under the action of spring. The innovation of the new sealing structure used by Tiangang United Special Steel lies in that a C-shaped leaf spring is installed between two adjacent springs, meanwhile a sloping plate is fixed on the inner side of the static oil plate. On the one hand, the sloping plate extends the sealing width of the static oil plate from $70 \mathrm{~mm}$ to $80 \mathrm{~mm}$, on the other hand, an oil groove inside the static oil plate and the movable oil plate is formed. The lubricating oil stored in the oil groove can guarantee that the contact surfaces of the static oil plate and the movable oil plate are fully lubricated, the wear is greatly reduced, and the sealing effect is improved, thereby reducing air leakage rate.

\subsection{Other Related Technologies}

\subsubsection{Steel Sludge Pretreatment}

Most sintering machines in China have digested some industrial solid wastes. ${ }^{24)}$ Although the valuable elements in solid waste can be employed efficiently, it brings a lot of trouble to granulation due to the special physical properties of some solid wastes. Tiangang United Special Steel adds some amount of converter sludge into the sintering materials to make use of iron (Total $\mathrm{Fe} \geq 50 \%$ ). Converter sludge is often formed into large pieces with poor granulation performance, influencing the contact of other sintering raw materials. Therefore, it is selected to pre-mix with the blast furnace return fines in sintering materials yard. As shown in Fig. 7, in the pretreatment process, the converter sludge is mixed with the blast furnace return fines firstly, then the mixture is transferred to a $5 \mathrm{~mm}$ sieve by belt. The part with size larger than $5 \mathrm{~mm}$ is transferred back to continue mixing, and the part with size smaller than $5 \mathrm{~mm}$ is sent to ingredients room. Uniformly dispersed sludge with a size less than
$5 \mathrm{~mm}$ is applied to participate in the batching effectively, which improves its granulation performance.

\subsubsection{Ignition Retrofit}

Sintering is a top-to-bottom process with the upper ignition and lower air extraction. The igniter is very important during the sintering process. Its combustion ability directly determines the generation of the binding phase and fuel consumption of sinter production. Existing igniters generally use multiple rows of nozzles to ignite on the materials surface vertically, however, parameters such as flame distribution, gas calorific value, and thermal insulation performance need to be further improved as the thickness of the materials layer further increasing.

As shown by position 4 in Figs. 3 and 8, the new igniter of Tiangang United Special Steel staggered the adjacent horizontal rows of fire spray groups horizontally, forming a dense spray fire point along the horizontal direction on the sintering materials surface, which eliminates the fire nozzle limitation of the size of the spray fire point gap (the distance between the spray point on the materials surface is less than $700 \mathrm{~mm}$ ). This improvement can effectively enhance the sintering effect of the raw materials and greatly save gas fuel.

\section{Production Indices and Benefits}

\subsection{Production Indices}

Since the implementation of the above-mentioned comprehensive technologies from the end of 2015, production throughout the whole year of 2019 has shown high stability, and the economic and technical indices have been greatly improved compared with that before implementation, among which the productivity has reached up to $1.89 \mathrm{t} /$ $\left(\mathrm{m}^{2} \cdot \mathrm{h}\right)$, solid fuel consumption and power consumption were only $41.85 \mathrm{~kg} / \mathrm{t}$ and $19.24 \mathrm{kWh} / \mathrm{t}$, respectively, all of which

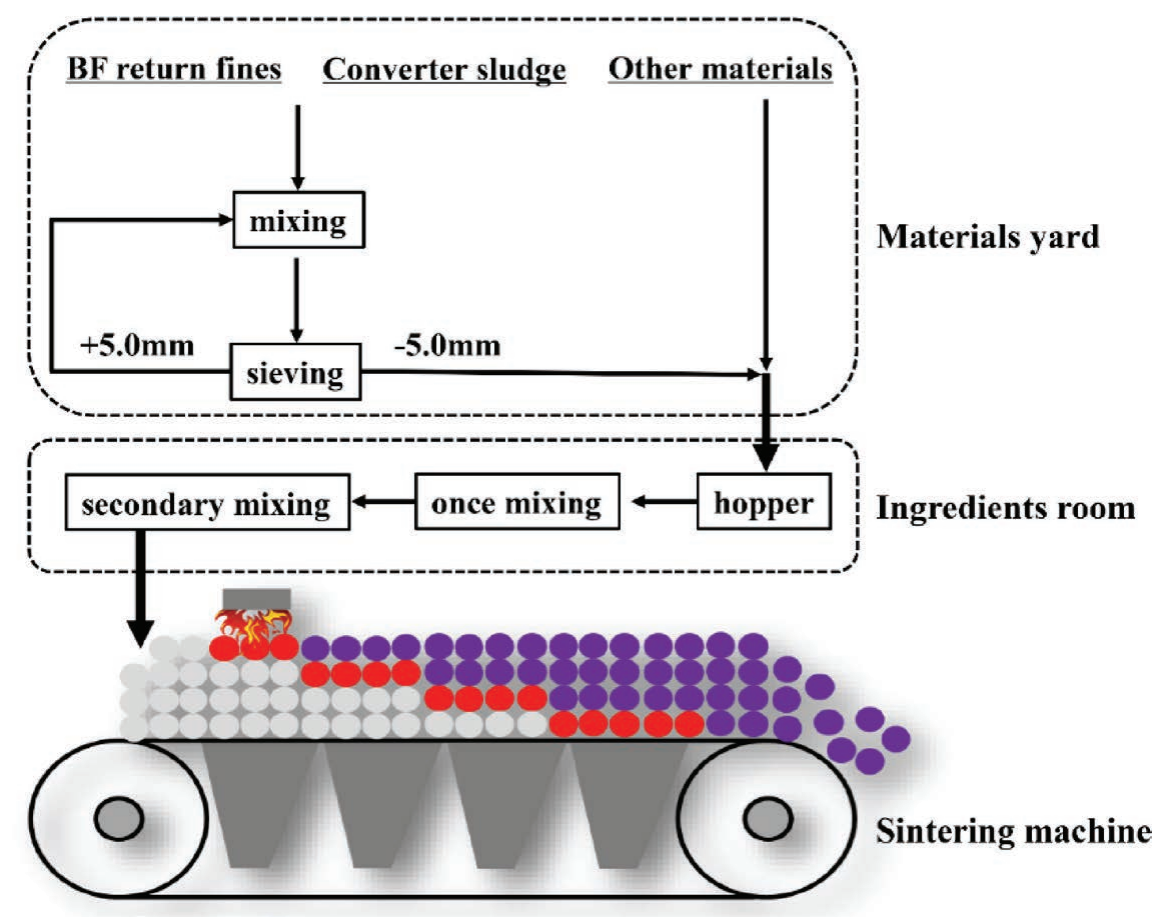

Fig. 7. Schematic diagram of converter sludge pretreatment process. (Online version in color.) 
are under industry-leading levels. ${ }^{25)}$

On the basis of a substantial increased output and a dramatic reduction in energy consumption, the chemical compositions, strength and metallurgical performance of the produced sinter meet the requirements of the 1000 $\mathrm{m}^{3}$ blast furnace supplied. The sinter quality in the vertical direction of the materials layer is uniform. Figure 9 shows the mineral phase structure, chemical compositions and metallurgical performance of the sinter upon the implementation of $1000 \mathrm{~mm}$ ultra-thick bed sintering. It can be noted that the main mineral phases inside the sinter are composite calcium ferrite (SFCA) and eroded

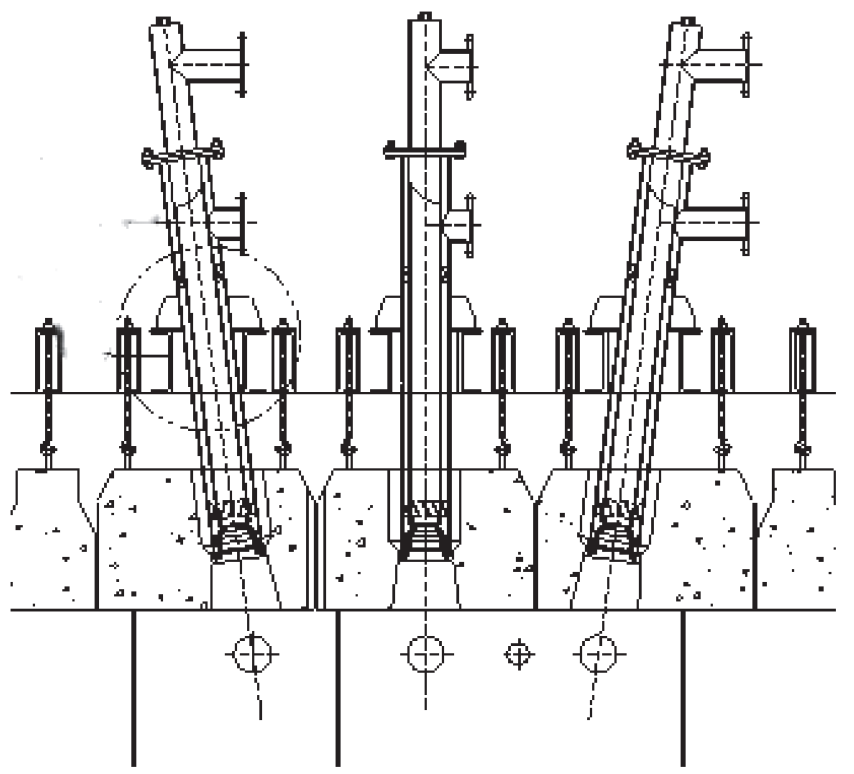

Fig. 8. Schematic diagram of ignition retrofit. hematite $\left(\mathrm{Fe}_{2} \mathrm{O}_{3}\right)$ grains under the high binary basicity of 2.12. The interweaving of the two forms a good micro composition of sinter. ${ }^{26-28)}$ The tumbler index (TI based on GB/T 8209-1987) and reduction index (RI based on $\mathrm{GB} / \mathrm{T} 13241-2017$ ) of the sinter are $78.24 \%$ and $87.17 \%$ respectively due to the excellent mineral composition, ${ }^{29}$ and the low-temperature reduction degradation index $\mathrm{RDI}_{+3.15}$ (RDI based on GB/T 13242-2017) also performs better than $72 \%$ recommended in the industry.

\subsection{Benefit Analysis}

As far as the two $230 \mathrm{~m}^{2}$ sintering machines of Tiangang United Special Steel are concerned, during the implementation of the $1000 \mathrm{~mm}$ ultra-thick bed sintering technologies, the economic benefits in increasing sinter production, reducing solid fuel consumption and power consumption are prominent although some costs from the equipment modification and testing. This demonstrates that net income is significant. In addition, the reduction in solid fuel consumption indicates less emissions of gaseous pollutants including carbon dioxide, nitrogen oxides, sulfur oxides, which leads to environment-friendly benefits.

Table 1. Comparison of economic and technical indices before and after implementation.

\begin{tabular}{ccc}
\hline Indices & $\begin{array}{c}\text { Before (2015 } \\
\text { annual average) }\end{array}$ & $\begin{array}{c}\text { After (2019 } \\
\text { annual average) }\end{array}$ \\
\hline Monthly output/ton & 378814 & 545467 \\
Productivity $\left./ \mathrm{t} / \mathrm{m}^{2} \cdot \mathrm{h}\right)$ & 1.38 & 1.89 \\
Solid fuel consumption $/ \mathrm{kg} / \mathrm{t}$ & 53.13 & 41.85 \\
Power consumption $/ \mathrm{kWh} / \mathrm{t}$ & 26.72 & 19.24 \\
Air leakage rate $/ \%$ & 55 & 34.68 \\
\hline
\end{tabular}

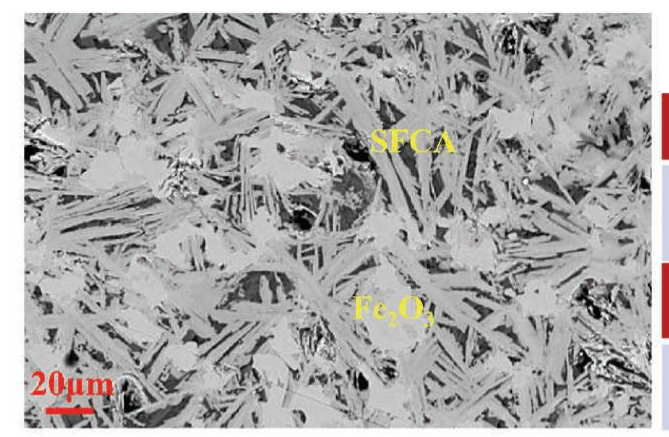

\section{Chemical compositions}

\begin{tabular}{|c|c|c|c|}
\hline $\mathrm{TFe}$ & $\mathrm{FeO}$ & $\mathrm{CaO}$ & $\mathrm{SiO}_{2}$ \\
\hline 55.31 & 9.01 & 11.50 & 5.43 \\
\hline $\mathrm{MgO}$ & $\mathrm{Al}_{2} \mathrm{O}_{3}$ & $\mathrm{Zn}$ & $\mathbf{R}_{2}$ \\
\hline 2.34 & 2.25 & 0.018 & 2.12 \\
\hline
\end{tabular}
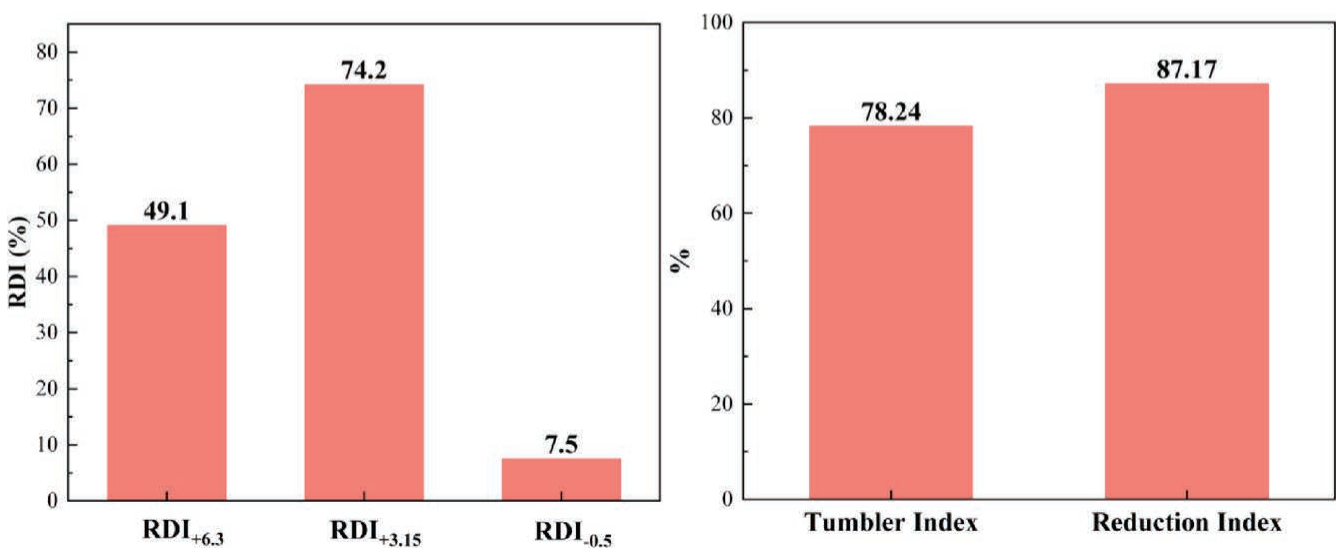

Fig. 9. Quality and metallurgical performance of sinter. (Online version in color.) 
According to sinter output of about 947 million tons and an average solid fuel consumption of $53 \mathrm{~kg} / \mathrm{t}$ in China in 2018. 11.36 million tons of solid fuel can be saved applied for all sintering machines in Hebei Province if the solid fuel consumption nationwide can reach the level of 1000 $\mathrm{mm}$ ultra-thick bed sintering in Tiangang United Special Steel, which has the largest steel output in China.

\section{Conclusions}

In this article, the production of $1000 \mathrm{~mm}$ ultra-thick bed sintering is introduced comprehensively in Tiangang United Special Steel in terms of background, technologies, and implementation effects. The main conclusions are as follows:

(1) Aiming at the characteristics of ultra-thick bed sintering, a triple sync theory including a liquid phase front, a heat transfer front, and a flame front was proposed. Based on this, "Full Active Lime Intensified sintering" technology focusing on the use of quicklime was developed.

(2) Based on technical concept, Tiangang United Special Steel implemented a number of comprehensive technologies including raw materials controls, granulation enhancements and air leakage improvements to achieve efficient and stable production with an ultra-thick bed sintering of $1000 \mathrm{~mm}$.

(3) The economic and technical indices of sintering and the sinter quality have reached advanced levels upon implementation. Among them, the productivity reaches 1.89 $\mathrm{t} /\left(\mathrm{m}^{2} \cdot \mathrm{h}\right)$; the solid fuel consumption is only $41.85 \mathrm{~kg} / \mathrm{t}$; the sintering power consumption is only $19.24 \mathrm{kWh} / \mathrm{t}$; the sinter tumbler index, the low temperature reduction degradation index $\mathrm{RDI}_{+3.15}$ and the reduction index are $78.24 \%, 74.2 \%$, $87.17 \%$ respectively. Comprehensive technologies bring significant economic and environmental benefits, providing a technical model for ultra-thick bed sintering.

\section{Acknowledgements}

The authors thank "Fundamental Research Funds for Central Universities" for financial support and Tiangang United Special Steel for permission to publish the work.

\section{REFERENCES}

1) R. R. Lovel, K. R. Vining and M. Dell'Amico: ISIJ Int., 49 (2009), 195.

2) Y. Liu, J. Yang, J. Wang, Z. L. Cheng and Q. W. Wang: Energy, 67 (2014), 370.

3) J. S. Feng, L. Zhao, S. Zhang and H. Dong: Appl. Therm. Eng., 175 (2020), 115370.

4) T. Jiang: The 12th Conf. China Iron and Steel, The Chinese Society for Metals, Beijing, (2019), 1 (in Chinese).

5) T. Umadevi, A. Brahmacharyulu, R. Sah and P. C. Mahapatra: Ironmaking Steelmaking, 41 (2014), 410.

6) T. Jiang, G. H. Li, H. T. Wang, K. C. Zhang and Y. B. Zhang: Ironmaking Steelmaking, 37 (2010), 1

7) T. Jiang, Z. W. Yu, Z. W. Peng, M. J. Rao, Y. B. Zhang and G. H. Li: ISIJ Int., 55 (2015), 1599.

8) G. H. Li, C. Liu, Z. W. Yu, M. J. Rao, Q. Zhong, Y. B. Zhang and T. Jiang: Energies, 11 (2018), 2382.

9) M. Zhou, Y. Wang, D. Zhao, J. Zhu, H. Liu, Q. Zhong, G. Li and T. Jiang: 11th Int. Symp. on High-Temperature Metallurgical Processing, Springer, Cham, (2020), 639.

10) K. Higuchi, T. Kawaguchi, M. Kobayashi, Y. Hosotani, K. Nakamura, K. Iwamoto and M. Fujimoto: ISIJ Int., 40 (2000), 1188.

11) Y. Z. Wang, J. L. Zhang, Z. J. Liu and C. B. Du: JOM, 69 (2017), 2404.

12) Y. Z. Wang, Z. J. Liu, J. L. Zhang, Y. P. Zhang, L. L. Niu and Q. Cheng: J. Clean. Prod., 252 (2020), 119855.

13) H. B. Zuo, J. L. Zhang, Z. Hu and T. J. Yang: Int. J. Miner. Metall. Mater., 20 (2013), 131.

14) C. E. Loo and M. F. Hutchens: ISIJ Int., 43 (2003), 630.

15) W. Yang, C. Ryu, S. Choi, E. Choi, D. Lee and W. Huh: ISIJ Int., 44 (2004), 492.

16) J. Mitterlehner, G. Loeffler, F. Winter, H. Hofbauer, H. Schmid, E. Zwittag, T. H. Buergler, O. Pammer and H. Stiasny: ISIJ Int., 44 (2004), 11.

17) B. G. Ellis, C. E. Loo and D. Witchard: Ironmaking Steelmaking, 34 (2007), 99.

18) N. A. S. Webster, M. I. Pownceby, R. Pattel, J. R. Manuel and J. A. Kimpton: ISIJ Int., 59 (2019), 263.

19) B. J. Shi, D. Q. Zhu, J. Pan, X. Q. Liu and S. W. Liu: Ironmaking Steelmaking, 47 (2020), 567.

20) S. Nomura, H. Ayukawa, H. Kitaguchi, T. Tahara, S. Matsuzaki, M. Naito, S. Koizumi, Y. Ogata, T. Nakayama and T. Abe: ISIJ Int., 45 (2005), 316.

21) K. J. Li, R. Khanna, J. L. Zhang, Z. J. Liu, V. Sahajwalla, T. J. Yang and D. Kong: Fuel, 133 (2014), 194.

22) J. D. Litster and A. G. Waters: Powder Technol., 62 (1990), 125.

23) X. L. Chen, Y. S. Huang, M. Gan, X. H. Fan, Z. Y. Yu and L. S. Yuan: J. Iron Steel Res. Int., 22 (2015),1107.

24) Y. Z. Wang, J. L. Zhang, Z. J. Liu, C. B. Du, J. Schenk, J. G. Shao and Y. P. Zhang: J. Clean. Prod., 234 (2019), 157

$25)$ D. J. Wang, S. L. Wu, C. X. Li and J. Zhu: ISIJ Int., 53 (2013), 1665.

26) N. A. S. Webster, M. I. Pownceby, I. C. Madsen and J. A. Kimpton: Metall. Mater. Trans. B, 43 (2012), 1344.

27) I. Tonžetić and A. Dippenaar: Miner. Eng., 24 (2011), 1258

28) Z. Wang, D. Pinson, S. Chew, B. J. Monaghan, M. Pownceby, N. A. S. Webster, H. Rogers and G. Q. Zhang: ISIJ Int., 56 (2016), 1138.

29) M. Sinha, S. H. Nistala, S. Chandra, T. R. Mankhand and A. K. Ghose: Ironmaking Steelmaking, 44 (2017), 100. 